\title{
COMPARATIVE ANALYSIS IN CONTINUOUS EXPANSION OF BOVINE AND HUMAN PRIMARY NUCLEUS PULPOSUS CELLS FOR TISSUE REPAIR APPLICATIONS.
}

\author{
D.H. Rosenzweig ${ }^{1,2, \S}$, J. Tremblay Gravel ${ }^{1, \S}$, D. Bisson ${ }^{1}$, J.A. Ouellet ${ }^{2}$, M.H. Weber ${ }^{2}$ and L. Haglund ${ }^{1,2, *}$ \\ ${ }^{1}$ The Orthopaedic Research Laboratory, Department of Surgery, McGill University, Montreal, QC, Canada \\ ${ }^{2}$ The McGill Scoliosis and Spine Research Group, Department of Surgery, McGill University, Montreal, QC, Canada \\ $\S$ These authors contributed equally
}

\begin{abstract}
Autologous NP cell implantation is a potential therapeutic avenue for intervertebral disc (IVD) degeneration. However, monolayer expansion of cells isolated from surgical samples may negatively impact matrix production by way of dedifferentiation. Previously, we have used a continuous expansion culture system to successfully preserve a chondrocyte phenotype. In this work, we hypothesised that continuous expansion culture could also preserve nucleus pulposus (NP) phenotype. We confirmed that serial passaging drove NP dedifferentiation by significantly decreasing collagen type II, aggrecan and chondroadherin (CHAD) gene expression, compared to freshly isolated cells. Proliferation, gene expression profile and matrix production in both culture conditions were compared using primary bovine NP cells. Both standard culture and continuous culture produced clinically relevant cell populations. However, continuous culture cells maintained significantly higher collagen type II, aggrecan and CHAD transcript expression levels. Also, continuous expansion cells generated greater amounts of proteoglycan, collagen type II and aggrecan protein deposition in pellet cultures. To our surprise, continuous expansion of human intervertebral disc cells - isolated from acute herniation tissue - produced less collagen type II, aggrecan and CHAD genes and proteins, compared to standard culture. Also, continuous culture of cells isolated from young non-degenerate tissue did not preserve gene and protein expression, compared to standard culture. These data indicated that primary bovine and human NP cells responded differently to continuous culture, where the positive effects observed for bovine cells did not translate to human cells. Therefore, caution must be exercised when choosing animal models and cell sources for pre-clinical studies.
\end{abstract}

Keywords: cell culture, intervertebral disc, nucleus pulposus cells, tissue engineering, elastic culture surfaces.

*Address for correspondence:

Lisbet Haglund

McGill University Health Centre, Department of Surgery

Montreal General Hospital, Room C10.148.2

1650 Cedar Ave, Montreal, QC H3G 1A4

Telephone: +15149341934 ext. 35380

Email: lisbet.haglund@mcgill.ca

\section{Introduction}

Intervertebral discs (IVDs) are the soft tissues between vertebral bones that serve to bear load and provide range of motion to the head and trunk. IVDs consist of a highly organised outer annulus fibrosus (AF), which resists tensile strains and encircles an inner gelatinous nucleus pulposus (NP) that serves to resist compressive forces (Adams et al., 2006). The disc is encased by hyaline cartilage endplates, which facilitate nutrient and waste diffusional exchange with the vasculature of vertebral bone. IVDs are mostly avascular, have very low cell density and a poor capacity for regeneration following injury and early degeneration (Liebscher et al., 2011; Maroudas et al., 1975). Degenerative disc disease (DDD) is characterised by an imbalance between matrix breakdown and synthesis, decreased proteoglycan and water content, increased cell death, inflammatory factors and protease activity, which all lead to mechanical failure and pain (Krock et al., 2016; Krock et al., 2015; Sato et al., 1999; Wuertz et al., 2013). Prevention of degeneration and matrix re-synthesis could be achieved by replenishment of the NP cell population through autologous cell implantation. Autologous chondrocyte implantation has had some success at repairing focal cartilage defects in patients (Brittberg et al., 1994; Brittberg et al., 2001). Autologous stem cell implantation for IVD repair has shown some clinical promise (Orozco et al., 2011). Autologous NP implantation has been shown to be safe and not cause low back pain, yet NP cells insufficiently repair IVDs (Mochida et al., 2015). Since they are already differentiated and programmed to secrete the appropriate matrix, newly injected NP cells should produce neo-matrix, replenish proteoglycan and water content and restore disc function. Current potential sources for primary NP cells include surgical herniated tissue and excised tissues from discectomy surgeries (Hegewald et al., 2011; Mochida et al., 2015). Biopsies and surgical samples typically contain an insufficient number of cells for immediate therapeutic impact and isolated cells must be expanded to generate clinically relevant cell populations to repair damaged tissues.

A major limiting factor in the implementation of this technique is maintaining an extracellular matrix (ECM) producing cell phenotype during the expansion. Standard monolayer culture of other cell types is known to lead to an altered protein production profile. Likely, dedifferentiation during the expansion process has negative impacts on the therapeutic effectiveness of cell implantation. This phenomenon has been well established for chondrocytes, 
where dedifferentiation decreases expression of collagen type II and aggrecan and increases expression of collagen type I (Darling et al., 2005; Lin et al., 2008). However, it is unclear if chondrocyte-like NP cells change in the same way during expansion (Kluba et al., 2005). Preserving maximal expression of ECM proteins in NP cells during culture is important to maximise the effectiveness of autologous cell implantation therapy in the NP of degenerate IVDs.

Standard monolayer culture of bovine and human chondrocytes is known to cause dedifferentiation though the interaction with rigid culture surfaces, enzymatic passaging of cells, increased proliferation and increased cell density (Darling et al., 2005; Homicz et al., 2002; Lefebvre et al., 1998; Lin et al., 2008). To circumvent this fundamental problem, we developed a culture method that reduces the need for passaging by growing the cells on a soft, continuously expanding culture surface (Majd et al., 2009). This method maintains high cell density on a soft, highly expandable silicone rubber culture dish. A motor and iris-like device slowly stretches the dish, continuously increasing the surface area to promote cell growth, while reducing contact inhibition and the need for enzymatic passaging. We have demonstrated that continuous expansion culture generates phenotypically superior articular chondrocytes for tissue regeneration applications (Rosenzweig et al., 2013; Rosenzweig et al., 2012a; Rosenzweig et al., 2012b); therefore, it could be equally effective for NP cell culture. We hypothesised that the continuous expansion device would maintain NP cell phenotype during expansion culture, as compared to standard culture. Initial experiments were performed with bovine NP cells to test and optimise the system, which was assessed by gene expression and followed by matrix protein assessment on pellet cultures. Finally, we tested this hypothesis using isolated disc cells from human disc herniation surgical samples and pure NP cells isolated from cadaveric organ donors with the goal of presenting an improved NP cell therapy application.

\section{Materials and Methods}

\section{Growth surface preparation}

For standard culture and continuous cultures, surfaces were treated as previously described (Rosenzweig et al., 2013; Rosenzweig et al., 2012a; Rosenzweig et al., 2012b). In the case of continuous expansion cultures, dishes entirely made of high extension silicone rubber (HESR) were used (Cytomec GmbH, Spiez, Switzerland). To facilitate cell adhesion, the hydrophobic silicone surface was chemically modified as previously described (Rosenzweig et al., 2012a). Briefly, plates were treated with $30 \%$ sulphuric acid for $15 \mathrm{~min}$; silanised with $1 \%$ (3 aminopropyl) triethoxysilane (APTES) (Sigma-Aldrich, Oakville, ON, Canada) for $2 \mathrm{~h}$ at $70{ }^{\circ} \mathrm{C}$; then surfaces were chemically functionalised using $6 \%$ glutaraldehyde for $15 \mathrm{~min}$. After each of these steps, dishes were rinsed three times with deionised water. Finally, dishes were sanitised in $70 \%$ ethanol for $10 \mathrm{~min}$ and then rinsed with sterile PBS. Plates were covered with $2 \mathrm{~mL}$ of $50 \mu \mathrm{g} / \mathrm{mL}$ rat tail collagen type I (Sigma) in PBS and incubated for $2 \mathrm{~h}$.

\section{Nucleus pulposus cell isolation}

NP cells were isolated from caudal discs of skeletally mature steer (18-21 months old). For each donor animal, cells were isolated from dissected NPs of two discs. Approximately $5 \mathrm{~g}$ of tissue were washed in sterile PBS supplemented with penicillin-streptomycin (pen/strep) and cut in a Petri dish into approximately $1 \mathrm{~mm}^{3}$ pieces. The dissected material was digested into a $50 \mathrm{~mL}$ conical tube, containing chondrocyte growth medium (high glucose Dulbecco's modified Eagle's medium (DMEM), $0.1 \mathrm{mM}$ nonessential amino acids, $10 \mathrm{mM}$ HEPES, $1 \mathrm{mM}$ sodium pyruvate, $10 \%$ foetal bovine serum (FBS), $1 \%$ pen/strep) supplemented with $1.5 \mathrm{mg} / \mathrm{mL}$ collagenase type II (Invitrogen/Gibco, Burlington, ON, Canada). Samples were incubated overnight at $37^{\circ} \mathrm{C}$ under constant agitation. Samples were run through $100 \mu \mathrm{m}$ filter (BD biosciences, Mississauga, ON, Canada) and centrifuged at $500 \times g$ for $5 \mathrm{~min}$. Supernatant was removed and the pelleted NP cells were resuspended in $10 \mathrm{~mL}$ chondrocyte growth medium and counted using a haemocytometer.

Surgical acute herniated human disc tissues were obtained from 3 donors (aged 41, 42 and 56) with consent and with institutional approval. Lumbar spine segments from 2 consented donors (ages 15 and 45) were obtained in collaboration with the provincially run organ donation programme, Transplant Quebec, with consent and institutional approval. Discs were excised from vertebral bone with a saw and NP tissue was macroscopically identified and dissected away from AF and inner AF tissue, as previously described (Gawri et al., 2014). In both cases, NP cell populations were isolated by enzymatic digestion in $0.04 \%$ collagenase type II in DMEM $110 \%$ FBS, $4.5 \mathrm{~g} / \mathrm{L}$ glucose, supplemented with $25 \mathrm{mMol} / \mathrm{L}$ HEPES, $0.25 \mu \mathrm{g} /$ $\mathrm{mL}$ amphotericin B, $2 \mathrm{mMol} / \mathrm{L}$ Gluta-MAX medium, $50 \mu \mathrm{g} / \mathrm{mL}$ of gentamicin sulphate (all from Gibco/Life Technologies, Burlington, ON, Canada) ] at $37^{\circ} \mathrm{C}$ overnight without agitation.

\section{Standard control cultures}

In standard cultures, NP cells were initially seeded in $35 \mathrm{~mm}$ dishes. Fig. 1 shows that at the time of starting the continuous culture protocol, standard culture cells were passaged ( $0.25 \%$ trypsin-EDTA solution, Invitrogen) to $55 \mathrm{~mm}$ dishes and cultured for $5 \mathrm{~d}$. Then, these cells were passaged to $100 \mathrm{~mm}$ dishes and cultured for an additional $5 \mathrm{~d}$ to match the timing and surface area of continuous culture cultures. The starting cell population for both, standard and continuous cultures, was $2.5 \times 10^{5}$ cells, which were cultured in chondrocyte growth medium. The initial seeding of cells in both cultures was 3 to $5 \mathrm{~d}$, to allow cell adhesion and spreading, with medium replaced every $3 \mathrm{~d}$. At the end of the 13 to $15 \mathrm{~d}$ expansion period, cells were either lysed using $1 \mathrm{~mL}$ of TRIzol reagent (Invitrogen) for RNA extraction or cultured in a pellet for histological purposes.

\section{Continuous expansion cultures}

The functionalised HESR dish was fastened to the expansion apparatus and stretched to $12.5 \mathrm{~cm}^{2}$, to reduce sagging of the surface and closely match the surface area of $35 \mathrm{~mm}$ dishes $\left(\sim 10 \mathrm{~cm}^{2}\right)$. After the initial 3 to $5 \mathrm{~d}$ 
period allocated for cell adhesion, the stretching program was activated, expanding the surface area of the dish to $76.8 \mathrm{~cm}^{2}$ over $10 \mathrm{~d}$, thereby matching the surface area of standard $100 \mathrm{~mm}$ culture dishes (Fig. 1). Medium was replaced every $3 \mathrm{~d}$. For the bovine experiments, individual experiments from 6 donor animals were completed with the exception of 4 donor animals for measuring CD24, KRT18 and KRT19 expression. After completion of the expansion, cells were counted using a haemocytometer. Their number was compared to the initial seeded number, to calculate the total number of colony doublings $\left(N_{D}\right)$ occurred. This was done using the following formula:

$$
N_{D}=\log _{2} \frac{N}{N_{0}}
$$

where $N$ is the final cell number and $N_{0}$ is the initial number of seeded cells (Matmati et al., 2013). Then, they were lysed using $1 \mathrm{~mL}$ of TRIzol reagent (Invitrogen) for RNA extraction (according to manufacturer's instructions) or cultured in a pellet for histological purposes.

\section{cDNA synthesis and quantitative real-time} polymerase chain reaction (qRT-PCR)

Following RNA extraction, 500 ng of total RNA was subjected to cDNA synthesis using the qScript cDNA synthesis kit, following the manufacturer's instructions (Quanta Biosciences, Gaithersburg, MD, USA) and standard recommended PCR protocols were performed in duplicate as previously described (Rosenzweig et al., 2012a). The average fold difference in gene expression of experimental samples compared with controls was calculated by the $2^{-\triangle \Delta C t}$ method, after normalising to GAPDH expression (Livak et al., 2001). PCR primers for major components of the NP extracellular matrix were used to determine that gene expression of key components was maintained. PCR primers for bovine collagen type II, aggrecan, Sox9, collagen type I, collagen type X and GAPDH were generated exactly as described elsewhere (Rosenzweig et al., 2013; Rosenzweig et al., 2012a; Rosenzweig et al., 2012b). Primers for bovine chondroadherin (CHAD), forward CTCAGTTCCCTGCAGCCCGGCGCTC and reverse CATGTTTCAGCGTGGTCACACCC were used as described by Tasheva et al. (2004). Bovine primers for CD24 were forward AGACTTACT CAAATCAAA and reverse AACAGTAGAGATGTAGAA (Gantenbein et al., 2014). Bovine primers for KRT18 and KRT19 were, respectively, forward TTGAGCTGCTCCATCTGCAT, reverse AAGGCCAGCTTGGAGAACAG and forward CGGTGCCACCATTGAGAACT, reverse CAAACTTGGTGCGGAAGTCA (Minogue et al., 2010). Human primers used for collagen type I, collagen type II and aggrecan were exactly as described in Aung et al. (2011).

\section{Pellet formation and culture}

Following expansion, 1 million cells from both culture techniques were pelleted in a $15 \mathrm{~mL}$ sterile conical tube and centrifuged at $500 \times g$ for $10 \mathrm{~min}$. Medium was aspirated and replaced with chondrogenic medium (DMEM high glucose with glutamine, $0.1 \mathrm{mM}$ nonessential amino acids, $10 \mathrm{mM}$ HEPES, $1 \mathrm{mM}$ sodium pyruvate, $1 \%$ pen/strep, $50 \mu \mathrm{g} / \mathrm{mL}$ ascorbic acid, 10nM dexamethasone and $1 \%$ insulin-transferrin-selenium [Gibco]). Then, cells were cultured for $21 \mathrm{~d}$ with medium changes every $3 \mathrm{~d}$.

\section{Preparation of cryosections}

Pellets were fixed in $4 \%$ paraformaldehyde (PFA) for $20 \mathrm{~min}$ at room temperature. Cryoprotection was performed by immersing pellets in a $10 \%$ sucrose solution in PBS for $30 \mathrm{~min}$ at $4{ }^{\circ} \mathrm{C}$. This was followed by immersion in a $20 \%$ sucrose solution in PBS at $4{ }^{\circ} \mathrm{C}$ for $2 \mathrm{~h}$. Finally, pellets were immersed in $30 \%$ sucrose in PBS at $4{ }^{\circ} \mathrm{C}$ overnight. Pellets were then embedded in Optimal Cutting Temperature (O.C.T) compound (Sakura Finetek USA, Torrance, CA, USA) in disposable embedding moulds (Ted-Pella, Warrington, PA, USA) and frozen at $-80{ }^{\circ} \mathrm{C}$. Frozen sections of $14 \mu \mathrm{m}$ thickness were cut using a Leica CM3050 S cryomicrotome, mounted on Superfrost + microscope slides (VWR) and stored at $-20{ }^{\circ} \mathrm{C}$.
A

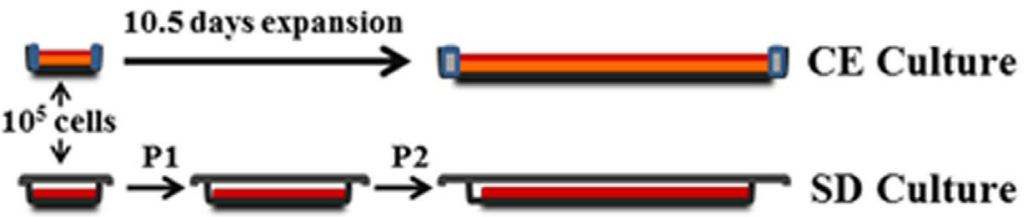

B

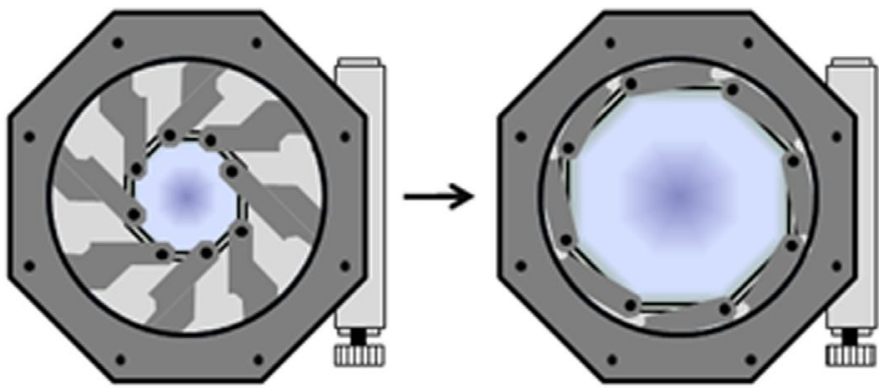

Fig. 1. Experimental design for comparative study of standard and continuous culture. (A) Equal number of primary NP cells was seeded on an HESR dish for continuous culture and a rigid polystyrene plate for standard culture. The continuous culture surface was continuously and evenly expanded from 12 to $76.8 \mathrm{~cm}^{2}$ over $10 \mathrm{~d}$. Simultaneously, cells in standard culture were passaged every 3 d. (B) Schematic illustration of the iris-like device used for continuous expansion of HESR dishes. 
Histological and immunofluorescence analyses

Since recent studies have suggested differential staining patterns for proteoglycan within NP tissue (Leung et al., 2009; Walter et al., 2015), pellets sections were stained with alcian blue and safranin $\mathrm{O} /$ fast green (all from Sigma) for proteoglycan and matrix structure visualisation. For immunofluorescence, pellet sections were blocked in permeabilisation buffer for $30 \mathrm{~min}$ (PBS, $0.1 \%$ Triton $\mathrm{X}-100$ and $1 \% \mathrm{BSA}$ ). Then, the permeabilised/blocked sections were incubated overnight at $4{ }^{\circ} \mathrm{C}$ with mouse monoclonal antibodies against collagen type I ( $1: 500$, M-38, Developmental Hybridoma Bank, University of Iowa, IA, USA) and aggrecan (1 : 200, 12/21/1-C-6, Developmental Studies Hybridoma Bank, University of Iowa) and rabbit polyclonal antibody against collagen type II ( $1: 500 ;$ ab34712, Abcam). The sections were washed 3 times in PBS and then incubated with either Alexa Fluor 488 Goat anti-Mouse IgG (1:250; Invitrogen) or Alexa Fluor 568 Goat anti-Rabbit IgG (1:250; Invitrogen) for $1 \mathrm{~h}$ at room temperature. Then, the sections were PBS washed, mounted with Fluoroshield with 4-,6-diamidino-2phenylindole (DAPI; Sigma) and visualised on an Olympus IX81 inverted fluorescence microscope. Morphological images were captured using a Zeiss Axiovert 40C microscope equipped with a Canon PowerShot A640 digital camera attached to a Zeiss MC80DX 1.0 tube adapter. Quantification of image pixels was performed. All images were processed in ImageJ software (National Institutes of Health, USA). Colour TIFF file images were converted to 32-bit images and inverted so that the background could be set to the lower threshold limit. After applying the image threshold, the background was removed and not counted toward mean pixel intensity. The exact same threshold limit was set for all images. Therefore, all holes or pockets within matrix of pellet sections were not counted within the pixel area. Mean pixel intensity and area were measured for the histological images due to the tears/holes within matrix of samples, while mean pixel intensity alone was measured for immunofluorescent images because holes/tears did not show.

\section{Statistical Analysis}

All statistical analyses were performed comparing continuous expansion cells or pellets to standard culture cells or pellets using paired $t$-tests from 4-6 independent experiments (noted in figure legends) using individual donors in each experiment. All $p$ values lower than 0.05 were considered to be statistically significant. Analyses
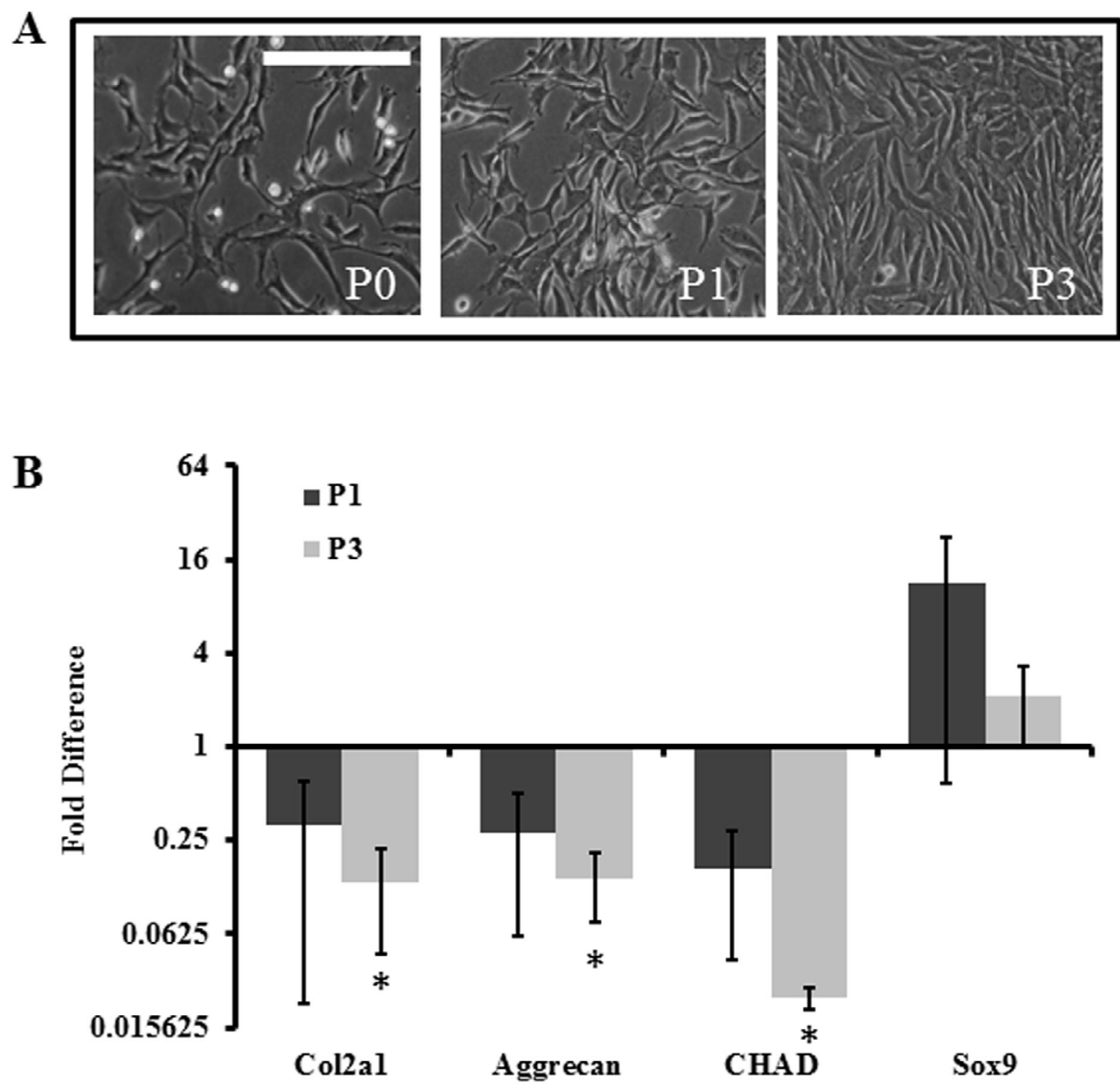

Fig. 2. Phenotype analysis of NP cells cultured over 3 passages on standard polystyrene dishes. (A) Gross morphology of primary NP cells in SD culture for 3 passages. Scale bar: $250 \mu \mathrm{m}$. (B) qRT-PCR revealed a pattern of progressive dedifferentiation from initial cell seeding to passage 3 in NP cells cultured in SD (standard culture method). Dark bars represent passage 1 and lighter bars represent passage 3 . Data are shown as mean $\pm \operatorname{SEM}(n=3)$. * indicates $p<0.05$. 
were performed using Graphpad Prism 6.0 (Graphpad Software, La Jolla, CA, USA).

\section{Results}

\section{Serial passaging of primary bovine NP cells led to} loss in ECM gene expression

To test for the effects of standard culture on gene expression, NP cells were cultured over three passages and gene expression was monitored. Cell morphology was similar between all passages (Fig.2A). Gene expression of the ECM components collagen type II, aggrecan and CHAD decreased with each passage number and was significantly decreased by $\mathrm{P} 3$ (passage 3 ) as compared to P0 (passage 0) controls (Fig. 2B). Expression of the master regulator of chondrogenesis, Sox9, showed no significant changes during passaging. This demonstrated that NP cells cultured by standard culture methods lost the expression of functionally important matrix molecules.

\section{Continuous expansion culture preserved gene} expression of ECM components in bovine NP cells

Our previous studies showed that continuous culture can preserve chondrocyte ECM production (Rosenzweig et al., 2013; Rosenzweig et al., 2012a; Rosenzweig et al., 2012b). In this study, we determined whether continuous culture had the same effect on NP cells. Bovine NP cells in continuous culture had a similar morphology to those in standard culture, as shown in Fig. 3A. To assess whether continuous culture can produce clinically relevant populations of NP cells, cell doubling was calculated. Similar population doubling was found using both methods and both total cell counts were suitable for clinical applications (Mochida et al., 2015; Orozco et al., 2011) (Fig. 3B). Continuous cultured cells displayed significantly higher expression of cytokeratin-18 (2.05 \pm 0.49 -fold difference, $p=0.003)$, collagen type II ( $3.71 \pm 1.44$-fold difference, $p=0.045)$ and aggrecan (5.32 \pm 2.08 -fold difference, $p=0.032)$ when compared to standard cultured cells (Fig. 3C). A trend for a difference was observed in cytokeratin-19 expression when compared to controls $(2.74 \pm 1.81$-fold difference, $p=0.052$ ). Also, continuous cultured cells displayed strong trends for higher CHAD (2.32 \pm 0.76 -fold difference, $p=0.057)$ and Sox9 (1.99 \pm 0.55 -fold difference, $p=0.051)$ expression, compared to standard cultured cells. Gene expression of CD24 was undetectable in all samples (not shown). There was no difference in collagen type I (2.05 \pm 0.49 -fold difference, $p=0.003)$ (Fig. 3C). To further determine preservation of NP phenotype, we assessed the aggrecan to collagen type II ratio. The ratio was approximately 3.31 times greater in continuous culture cells compared to standard cultures $(p=0.041)$. These data strongly indicated that continuous culture preserved gene expression of ECM components in bovine NP cells.

\section{Continuous culture enhances protein expression of ECM components in bovine NP cells}

To assess the potential of cultured NP cells to generate a NP-like tissue, continuous and standard cultured cells
A

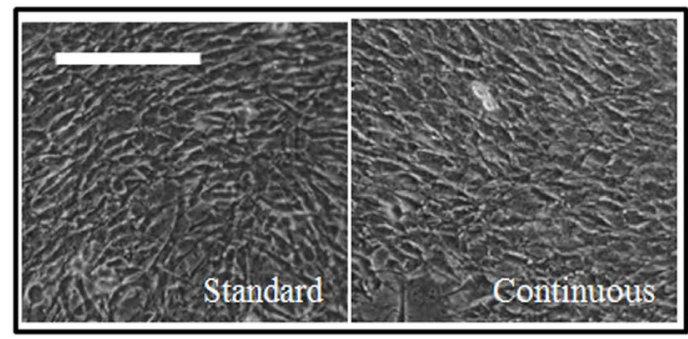

B

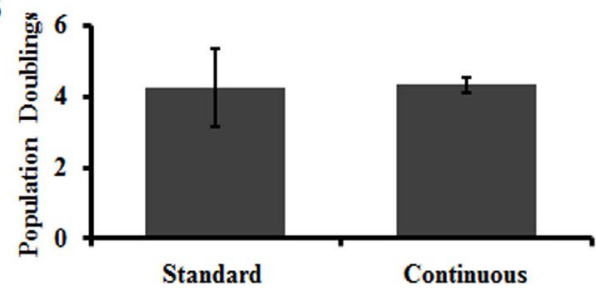

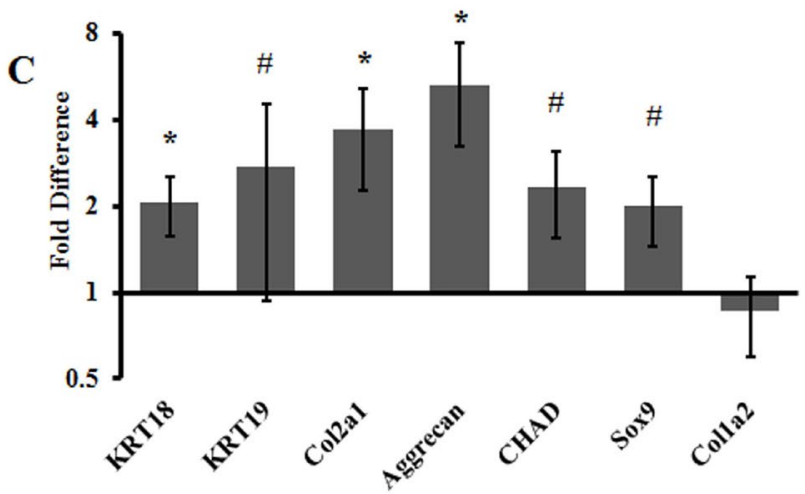

D

Relative Aggrecan:Collagen II Expression

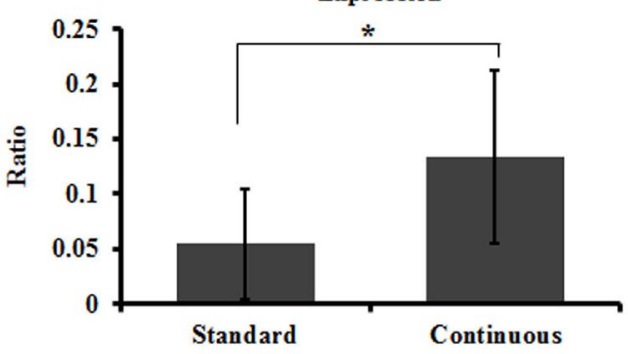

Fig. 3. Phenotype comparison of standard and continuous cultured NP cells. (A) Gross morphology of NP cells in standard and continuous culture. Scale bar: $250 \mu \mathrm{m}$. (B) Cells were counted after completion of the culture protocol and doublings were calculated. (C) qRT-PCR was performed in duplicate by comparing gene expression in continuous culture with standard culture. Data are shown as mean $\pm \mathrm{SEM}, n=6$ ( $n=4$ for KRT18 and KRT19). (D) Aggrecan : Collagen type II ratio was calculated based on relative expressions. Data are shown as mean $\pm \mathrm{SD}$, $n=6$. Statistical significance was determined by paired Student's $t$-test where $* p<0.05$ and $\# p<0.06$. 
were centrifuged to form three-dimensional (3D) pellet cultures and submerged in chondrogenic medium. Histological analysis showed that pellets from continuous cultured cells contained dramatically more sulphated GAG compared with pellets from standard cultures, as evident by alcian blue and safranin $\mathrm{O} /$ fast green staining (Fig.4, upper panels). Quantification of mean pixel intensity of these stained sections showed significantly $(p=0.024)$ more alcian blue stain in pellets from continuous culture $(5163 \pm 310)$ compared to standard culture pellets (3671 \pm 655$)$ (Fig. 4, upper right graph). Similarly, a strong trend with enhanced safranin-O staining was observed in continuous versus standard culture pellets $(6583 \pm 1160$; $3821 \pm 2125, p=0.051$ ). As shown in Fig. 4 (lower panels), immunofluorescence analysis revealed that continuous pellets contained stronger collagen type II and aggrecan signals as compared to standard pellets. Quantification of pixel intensity revealed a moderate increase in collagen type II signal and a significantly higher aggrecan protein deposition in continuous culture pellets $(34.3 \pm 2.49)$ versus standard culture pellets $(24.1 \pm 4.83)(p=0.031$, Fig. 4, lower right graph). There was no visible difference in staining for collagen type I. Taken together, these data indicated that NP cells from continuous culture had a stronger potential to produce the major components of the NP extracellular matrix.

\section{Continuous expansion did not preserve gene expression of ECM components in human intervertebral disc cells}

A potential source for autologous disc cell implantation is cells isolated from acute herniation tissue, which presents a mix of NP and AF cells. To assess whether the positive effects of continuous culture observed for bovine NP cells translated to human intervertebral disc cells, NP ECM gene expression and protein deposition in pellet cultures was compared between continuous and standard cultured of human disc cells isolated from acute herniation. Continuous cultured cells showed similar morphology and cells doubling compared to standard culture in herniated disc cells (Fig. 5A,B). Continuous culture cells showed no change in collagen type II or collagen type I gene expression $(0.72 \pm 0.45$ fold difference and $1.04 \pm 0.48$ fold difference, respectively) compared to standard
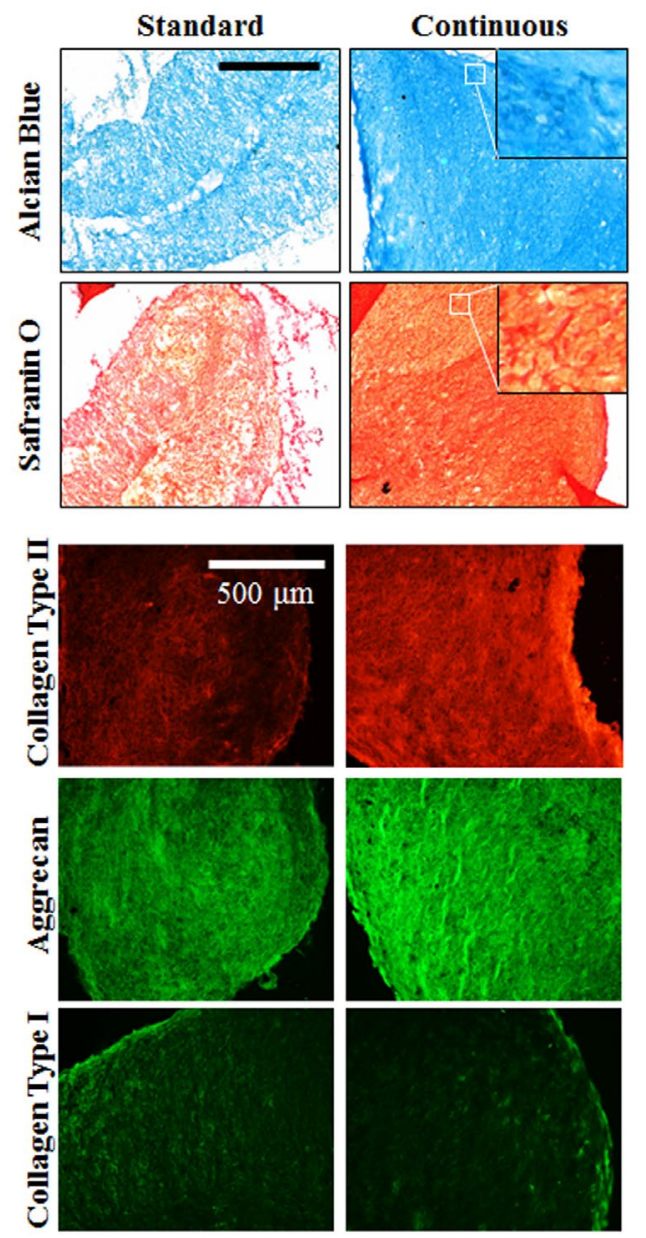
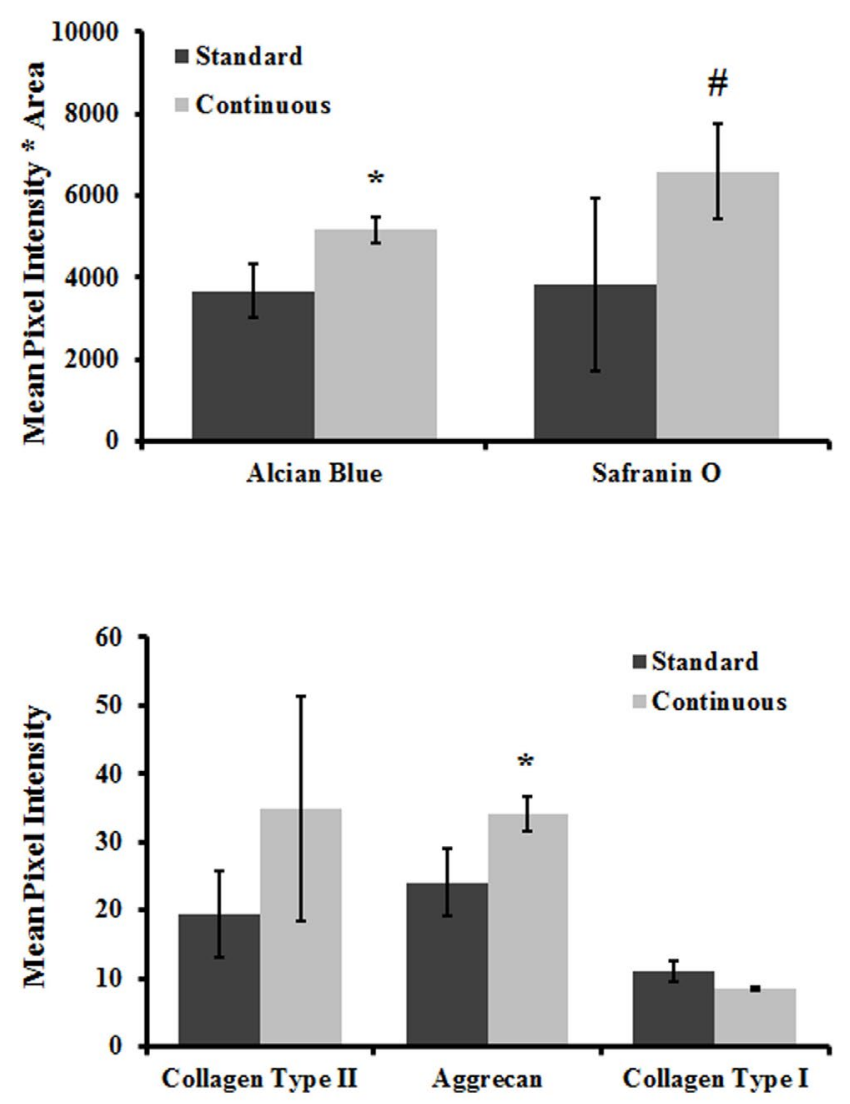

Fig. 4. NP-like matrix deposition in re-differentiated NP cells. Upper panels, continuous and standard cultured NP cells in re-differentiated 3D pellet culture with chondrogenic medium. From top, first row (blue) alcian blue and second row (red) safranin $\mathrm{O}$ /fast green staining of frozen sections of pellets. Representative images shown, $n=4$. Bottom panels, immunofluorescence microscopy probing for collagen type II (top row, red), aggrecan (middle row, green) and Collagen Type I (bottom row, green) was performed on frozen sections of pellets. Representative images shown, $n=4$. 
culture cells (Fig. 5C). However, continuous culture cells displayed significantly reduced aggrecan gene expression $(0.46 \pm 0.29$ fold difference, $p=0.016)$ compared to standard culture cells. Pellet cultures from continuous culture herniated disc cells showed less proteoglycan staining and no difference in collagen type II protein deposition (Fig. 5D), reflecting the gene expression results. These data indicated that continuous culture of isolated herniated disc cells did not preserve or enhance ECM gene and protein expression.

To rule out the possibility that herniated disc cells were already influenced by tissue degeneration and dedifferentiated, pure populations of human NP cells were isolated from non-degenerate NP tissue of organ donors. Isolated cells were subjected to continuous and standard culture expansion. Surprisingly, compared to standard culture, continuous culture had decreased collagen type II ( 0.58 fold difference), aggrecan ( 0.157 fold difference $)$ and collagen type I ( 0.815 fold difference) gene expression in NP cells isolated from a 15-year old donor (Fig. 6A). In a 45-year old donor, continuous culture had increased collagen type II expression (15.1 fold difference), decreased collagen type I expression ( 0.67 fold difference) and not affected aggrecan gene expression (Fig. 6B). Additionally, pellet cultures from both donors revealed no major differences in proteoglycan or collagen type II protein deposition between continuous or standard culture cell (Fig. 6C,D).

\section{Discussion}

The potential of using autologous NP cells from acute herniated or surgically excised NP tissue from patients undergoing vertebral fusions is an attractive means for potential prevention of disc degeneration in adjacent segments (Mochida et al., 2015). However, the number of cells retrieved from herniated tissue is low and expansion would be necessary to yield enough cells for supplementation therapy. In the present study, we evaluated if continuous expansion culture could be a way to produce clinically relevant cell populations with preserved matrix production. Bovine cells and tissue are used in many studies focused on IVD regeneration, since they are readily available and are similar in shape and size to humans. Therefore, we started our investigation using bovine cells. Primary bovine NP cells rapidly lost ECM gene expression in standard serial passaging, which is consistent with results observed in chondrocytes, MSCs and human NP cells (Darling et al., 2005; Kluba et al., 2005; Majd et al., 2011). This was demonstrated by decreased gene expression of collagen type II, aggrecan and CHAD and increased expression of collagen type I, previously associated with loss of chondrogenic phenotype (Rosenzweig et al., 2013; Rosenzweig et al., 2012a; Rosenzweig et al., 2012b). In contrast, continuous culture preserved gene expression of the NP-associated ECM components KRT18, KRT19, collagen type II, aggrecan
A

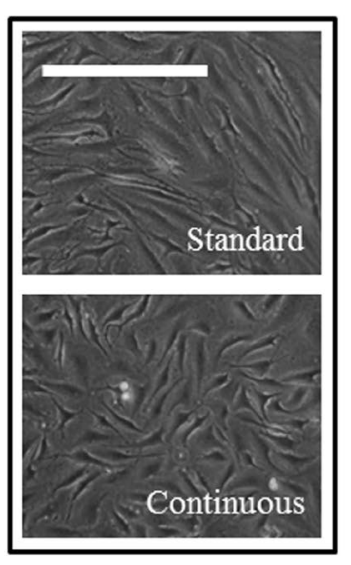

B

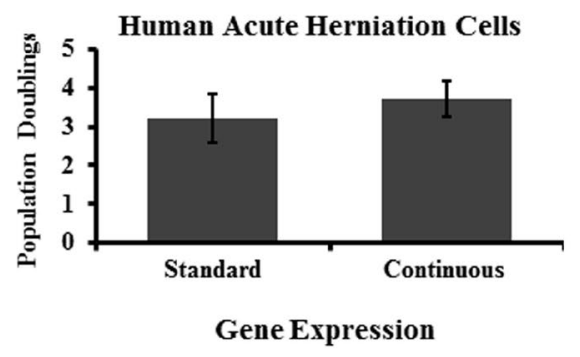

C

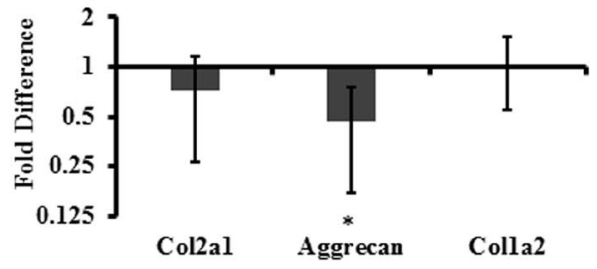

D

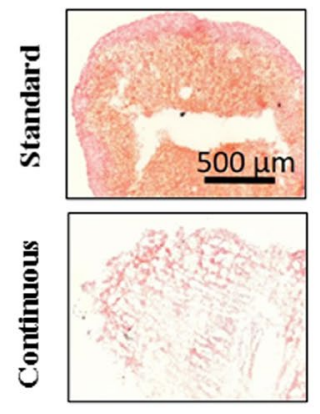

Safranin 0
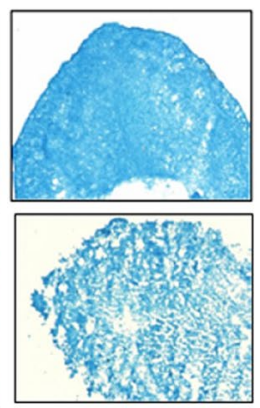

Alcian Blue

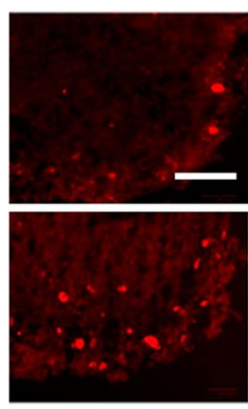

Collagen Type II
Fig. 5. Continuous culture of human disc cells isolated from acute herniations. (A) Representative images of standard and continuous cultured human disc herniation cells. Scale bar: $200 \mu \mathrm{m}$. (B) Quantification of population doublings. Data are shown as mean $\pm \mathrm{SD}, n=3$. (C) Gene expression analysis comparing continuous culture cells to standard culture. Data are shown as mean $\pm \mathrm{SD}, n=3$. * indicates $p<0.05$. (D) Safranin O and alcian blue staining comparing proteoglycan deposition, scale bar: $500 \mu \mathrm{m}$. Collagen type II immunofluorescence (red) indicating protein deposition. 
and CHAD and enhanced the cells regenerative capacity, when subjected to pellet culture. These results were very encouraging and, if translatable to human cells, would be highly relevant to NP tissue regeneration purposes.

We were able to evaluate human NP cells from clinically relevant herniated NP tissue. However, in this case, continuous expansion neither preserved gene expression of the ECM components collagen type II, aggrecan and CHAD, nor enhanced the regenerative capacity, when subjected to pellet culture. In fact continuous expansion resulted in lower ECM gene expression and inferior matrix production when subjected to pellet culture. Quite likely, cells extracted from hernia tissue are a mixed population of nucleus pulposus, annulus fibrosus and other various cell types from the external periphery (Brock et al., 1992; Harada et al., 1989; Willburger et al., 2004). During the period preceding hernia resection, this tissue will experience unusual loading conditions and potential inflammatory responses (Ikeda et al., 1996; Park et al., 2001). The increased inflammatory factors may have highly negative or fibrotic influence on the phenotype of the cells isolated from this herniation tissue, reflecting markers of degeneration. Indeed, we have previously established the negative effects of low frequency, high magnitude stretch on healthy NP and AF cells (Gawri et al., 2014). Perhaps, human cells are more sensitive to mechanical stretch than bovine NP cells, as opposed to being less sensitive to mechanical compression, where human discs can survive higher loads (Haglund et al., 2011; Rosenzweig et al., 2016). In conclusion, herniated human cells under continuous expansion promoted an expression profile less representative of nucleus pulposus cells, indicating that standard culture methods were more suitable since standard culture pellets showed better ECM deposition.

There were several differences between the human herniation samples and the bovine NP tissue used in this study. First, the differences may be due to age, since herniation cells were isolated from adults and the bovine cells were from young animals. Also, there are inherent differences between human and bovine NP cells that could play a role in the differentiation status. Although the human lumbar disc and the bovine caudal disc are similar in many aspects, some significant differences exist between the two species (Haglund et al., 2011; Jim et al., 2011). Cell density was higher in the young bovine caudal disc than in the older human lumbar discs from which herniations
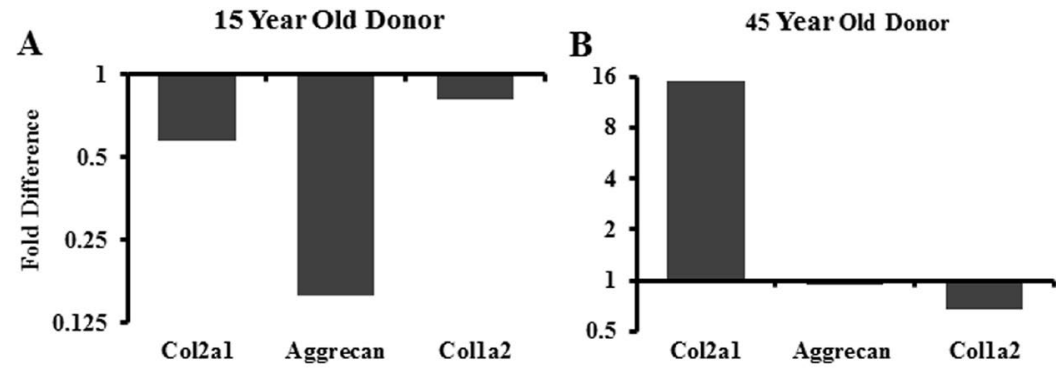

C
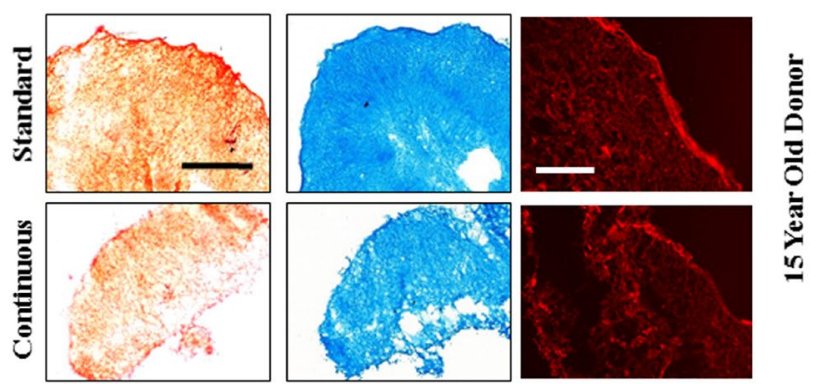

D

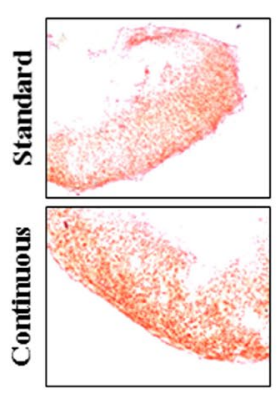

Safranin 0

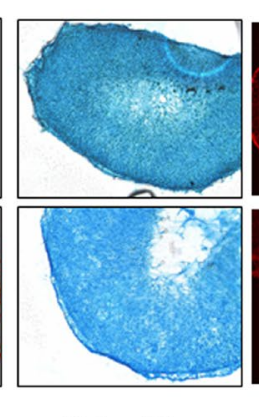

Alcian Blue

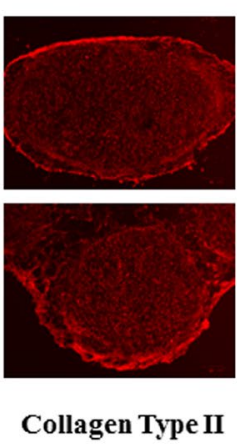

Fig. 6. Continuous culture of human NP cells isolated from young and older non-degenerate, pure NP tissue. Gene expression analysis in of collagen type II, aggrecan and collagen type I (A,B). Safranin $\mathrm{O}$ and alcian blue staining comparing proteoglycan deposition, scale bar: $500 \mu \mathrm{m}$. Collagen type II immunofluorescence (red) indicating protein deposition $(\mathbf{C}, \mathbf{D})$. 
were extracted. To address this, we performed continuous expansion of pure NP cells isolated from a 15 year-old donor. Even in this young donor, continuous expansion did not retain or improve the NP gene expression or matrix deposition.

To rule out the idea that the herniation cells were already predisposed to a more fibrotic condition, we performed continuous expansion of pure NP cells, isolated from a healthy donor of similar age to the herniated samples. In this case as well, continuous culture gave a reduced aggrecan production, whereas an improvement in collagen type II expression was seen at the gene level. Although these non-degenerate experiments were based only on 2 donors, continuous expansion of the non-degenerate human NP cells showed a variable response, seemingly not superior to standard culture.

Cells isolated from herniation may still be a suitable starting material in autologous cell implantation for disc therapy (Hegewald et al., 2011). It would be clinically valuable to use the mixed herniated cell source, since attempting to extract and use only NP cells out of the mixed population would be quite difficult because cell surface markers that reliably differentiate nucleus pulposus cells from annulus fibrosus and cartilaginous endplate cells have yet to been identified (Thorpe et al., 2016). Taken together, it is evident that continuous expansion of human disc cells offers little advantage over standard culture.

In this study, both bovine and human experiments were conducted under normoxic conditions $\left(20 \% \mathrm{O}_{2}\right)$ in standard, high glucose $\mathrm{pH} 7.2$ culture medium; a condition that most likely favours cells from the young bovine discs. All cells were cultured under high glucose conditions, which also may have favoured bovine NP cells, since high glucose increased ECM matrix gene expression in bovine NP cells more than in human NP cells (Rinkler et $a l ., 2010)$. It has been previously reported that culturing bovine NP cells at more physiological $\mathrm{pH}$ of 6.5 enhances their matrix metabolic activity (Neidlinger-Wilke et al., 2012). It is possible that human NP cells are even more sensitive to being cultured at higher $\mathrm{pH}$ than bovine cells, which would further slow their ECM production. Assessing all of the effects of different physiological parameters of the disc microenvironment are required to evaluate the regenerative potential of in vitro expanded human NP cells. Ultimately, caution must be exercised when interpreting results of animal studies in terms of possible impact on human health.

\section{Conclusions}

Autologous cell implantation for disc repair and inhibition of degeneration is becoming a clinical reality and, therefore, a sufficient number of optimally cultured cells are required to be of therapeutic value. This study demonstrated that standard monolayer culturing of primary bovine NP cells on polystyrene dishes resulted in a loss in gene expression of important matrix molecules. However, continuous culture of bovine NP cells prevented this loss while obtaining clinically relevant cell populations. The continuous cultured cells demonstrated an improved capacity for tissue regeneration in 3D pellets. These results were not recapitulated when using isolated human disc cells from either surgical herniated tissue or healthy non-degenerate tissues. Therefore, caution should be exercised when interpreting results from animal studies in comparison to human application.

\section{Acknowledgements}

We thank Dr Peter J. Roughley for all discussions, comments and suggestions. This work was financially supported by the Canadian Institutes of Health Research (CIHR) grant CIHR MOP-119564, AOSpine, SRN_2011_04_10498 to LH and AO Spine Research Network Consortium Award 2014. Histological sectioning, staining and imaging were aided by the FRQS-sponsored RSBO. DHR and LH designed the study. DHR, JTG and DB completed the experimental work and analysed the data. DHR, JTG, DB drafted the manuscript. MHW and JAO obtained surgical samples for human experiments. MHW, JAO and LH critically reviewed the work. All authors were involved in the experimental design and editing and revising of the manuscript. All authors approved the final version.

\section{References}

Adams MA, Roughley PJ (2006) What is intervertebral disc degeneration, and what causes it? Spine (Phila $\mathrm{Pa}$ 1976) 31: 2151-2161.

Aung A, Gupta G, Majid G, Varghese S (2011) Osteoarthritic chondrocyte-secreted morphogens induce chondrogenic differentiation of human mesenchymal stem cells. Arthritis Rheum 63: 148-158.

Brittberg M, Lindahl A, Nilsson A, Ohlsson C, Isaksson O, Peterson L (1994) Treatment of deep cartilage defects in the knee with autologous chondrocyte transplantation. N Engl J Med 331: 889-895.

Brittberg M, Tallheden T, Sjogren-Jansson B, Lindahl A, Peterson L (2001) Autologous chondrocytes used for articular cartilage repair: An update. Clin Orthop Relat Res S337-348.

Brock M, Patt S, Mayer HM (1992) The form and structure of the extruded disc. Spine (Phila Pa 1976) 17: 1457-1461.

Darling EM, Athanasiou KA (2005) Rapid phenotypic changes in passaged articular chondrocyte subpopulations. J Orthop Res 23: 425-432.

Gantenbein B, Calandriello E, Wuertz-Kozak K, Benneker LM, Keel MJ, Chan SC (2014) Activation of intervertebral disc cells by co-culture with notochordal cells, conditioned medium and hypoxia. BMC Musculoskelet Disord 15: 422.

Gawri R, Rosenzweig DH, Krock E, Ouellet JA, Stone LS, Quinn TM, Haglund L (2014) High mechanical strain of primary intervertebral disc cells promotes secretion of inflammatory factors associated with disc degeneration and pain. Arthritis Res Ther 16: R21.

Haglund L, Moir J, Beckman L, Mulligan KR, Jim B, Ouellet JA, Roughley P, Steffen T (2011) Development 
of a bioreactor for axially loaded intervertebral disc organ culture. Tissue Eng Part C Methods 17: 1011-1019.

Harada Y, Nakahara S (1989) A pathologic study of lumbar disc herniation in the elderly. Spine (Phila Pa 1976) 14: 1020-1024.

Hegewald AA, Endres M, Abbushi A, Cabraja M, Woiciechowsky C, Schmieder K, Kaps C, Thome C (2011) Adequacy of herniated disc tissue as a cell source for nucleus pulposus regeneration. J Neurosurg Spine 14: 273-280.

Homicz MR, Schumacher BL, Sah RL, Watson D (2002) Effects of serial expansion of septal chondrocytes on tissue-engineered neocartilage composition. Otolaryngol Head Neck Surg 127: 398-408.

Ikeda T, Nakamura T, Kikuchi T, Umeda S, Senda H, Takagi K (1996) Pathomechanism of spontaneous regression of the herniated lumbar disc: histologic and immunohistochemical study. J Spinal Disord 9: 136-140.

Jim B, Steffen T, Moir J, Roughley P, Haglund L (2011) Development of an intact intervertebral disc organ culture system in which degeneration can be induced as a prelude to studying repair potential. Eur Spine J 20: 1244-1254.

Kluba T, Niemeyer T, Gaissmaier C, Grunder T (2005) Human anulus fibrosis and nucleus pulposus cells of the intervertebral disc: effect of degeneration and culture system on cell phenotype. Spine (Phila Pa 1976) 30: 2743 2748.

Krock E, Currie JB, Weber MH, Ouellet JA, Stone LS, Rosenzweig DH, Haglund L (2016) Nerve growth factor is regulated by toll-like receptor 2 in human intervertebral discs. J Biol Chem 291: 3541-3551.

Krock E, Rosenzweig DH, Haglund L (2015) The inflammatory milieu of the degenerate disc: is mesenchymal stem cell-based therapy for intervertebral disc repair a feasible approach? Curr Stem Cell Res Ther 10: $317-328$.

Lefebvre V, de Crombrugghe B (1998) Toward understanding sox 9 function in chondrocyte differentiation. Matrix Biol 16: 529-540.

Leung VY, Chan WC, Hung SC, Cheung KM, Chan D (2009) Matrix remodeling during intervertebral disc growth and degeneration detected by multichromatic fast staining. J Histochem Cytochem 57: 249-256.

Liebscher T, Haefeli M, Wuertz K, Nerlich AG, Boos N (2011) Age-related variation in cell density of human lumbar intervertebral disc. Spine (Phila Pa 1976) 36: 153159.

Lin Z, Fitzgerald JB, Xu J, Willers C, Wood D, Grodzinsky AJ, Zheng MH (2008) Gene expression profiles of human chondrocytes during passaged monolayer cultivation. J Orthop Res 26: 1230-1237.

Livak KJ, Schmittgen TD (2001) Analysis of relative gene expression data using real-time quantitative pcr and the 2(-delta delta $\mathrm{c}(\mathrm{t})$ ) method. Methods 25: 402-408.

Majd H, Quinn TM, Wipff PJ, Hinz B (2011) Dynamic expansion culture for mesenchymal stem cells. Methods Mol Biol 698: 175-188.

Majd H, Wipff PJ, Buscemi L, Bueno M, Vonwil D, Quinn TM, Hinz B (2009) A novel method of dynamic culture surface expansion improves mesenchymal stem cell proliferation and phenotype. Stem Cells 27: 200-209.
Maroudas A, Stockwell RA, Nachemson A, Urban J (1975) Factors involved in the nutrition of the human lumbar intervertebral disc: cellularity and diffusion of glucose in vitro. Journal of Anatomy 120: 113-130.

Matmati M, Ng TF, Rosenzweig DH, Quinn TM (2013) Protection of bovine chondrocyte phenotype by heat inactivation of allogeneic serum in monolayer expansion cultures. Ann Biomed Eng 41: 894-903.

Minogue BM, Richardson SM, Zeef LA, Freemont AJ, Hoyland JA (2010) Transcriptional profiling of bovine intervertebral disc cells: implications for identification of normal and degenerate human intervertebral disc cell phenotypes. Arthritis Res Ther 12: R22.

Mochida J, Sakai D, Nakamura Y, Watanabe T, Yamamoto Y, Kato S (2015) Intervertebral disc repair with activated nucleus pulposus cell transplantation: a three-year, prospective clinical study of its safety. Eur Cell Mater 29: 202-212.

Neidlinger-Wilke C, Mietsch A, Rinkler C, Wilke HJ, Ignatius A, Urban J (2012) Interactions of environmental conditions and mechanical loads have influence on matrix turnover by nucleus pulposus cells. J Orthop Res 30: 112121.

Orozco L, Soler R, Morera C, Alberca M, Sanchez A, Garcia-Sancho J (2011) Intervertebral disc repair by autologous mesenchymal bone marrow cells: a pilot study. Transplantation 92: 822-828.

Park JB, Chang H, Lee JK (2001) Quantitative analysis of transforming growth factor-beta 1 in ligamentum flavum of lumbar spinal stenosis and disc herniation. Spine (Phila Pa 1976) 26: E492-495.

Rinkler C, Heuer F, Pedro MT, Mauer UM, Ignatius A, Neidlinger-Wilke C (2010) Influence of low glucose supply on the regulation of gene expression by nucleus pulposus cells and their responsiveness to mechanical loading. J Neurosurg Spine 13: 535-542.

Rosenzweig DH, Chicatun F, Nazhat SN, Quinn TM (2013) Cartilaginous constructs using primary chondrocytes from continuous expansion culture seeded in dense collagen gels. Acta Biomater 9: 9360-9369.

Rosenzweig DH, Gawri R, Moir J, Beckman L, Eglin D, Steffen T, Roughley PJ, Ouellet JA, Haglund L (2016) Dynamic loading, matrix maintenance and cell injection therapy of human intervertebral discs cultured in a bioreactor. Eur Cell Mater 30: 26-39.

Rosenzweig DH, Matmati M, Khayat G, Chaudhry S, Hinz B, Quinn TM (2012a) Culture of primary bovine chondrocytes on a continuously expanding surface inhibits dedifferentiation. Tissue Eng Part A 18: 2466-2476.

Rosenzweig DH, Solar-Cafaggi S, Quinn TM (2012b) Functionalization of dynamic culture surfaces with a cartilage extracellular matrix extract enhances chondrocyte phenotype against dedifferentiation. Acta Biomater 8: 3333-3341.

Sato K, Kikuchi S, Yonezawa T (1999) In vivo intradiscal pressure measurement in healthy individuals and in patients with ongoing back problems. Spine (Phila Pa 1976) 24: 2468-2474.

Tasheva ES, Klocke B, Conrad GW (2004) Analysis of transcriptional regulation of the small leucine rich proteoglycans. Mol Vis 10: 758-772. 
Thorpe AA, Binch AL, Creemers LB, Sammon C, Le Maitre CL (2016) Nucleus pulposus phenotypic markers to determine stem cell differentiation: fact or fiction? Oncotarget 7: 2189-2200.

Walter BA, Torre OM, Laudier D, Naidich TP, Hecht AC, Iatridis JC (2015) Form and function of the intervertebral disc in health and disease: a morphological and stain comparison study. J Anat 227: 707-716.

Willburger RE, Ehiosun UK, Kuhnen C, Kramer J, Schmid G (2004) Clinical symptoms in lumbar disc herniations and their correlation to the histological composition of the extruded disc material. Spine (Phila Pa 1976) 29: 1655-1661.

Wuertz K, Haglund L (2013) Inflammatory mediators in intervertebral disk degeneration and discogenic pain. Global Spine J 3: 175-184.

\section{Discussion with Reviewers}

Benjamin Gantenbein: Can you elaborate on the general clinical relevance of expanding cells without the need to passage them? What do you think is more important: improving media formulations, to make them free of animal compounds, expanding cells without passaging steps or maybe a combination of both? With respect to clinical relevance, can you compare this iris-like approach, to increase cell surface, with recent cell-layer based thermoreversible pNIPAM technology, to lift-off cells from culture dishes?

Authors: The clinical relevance of expanding cells without passaging is twofold. The ability to expand cells without standard passaging abolishes cell enzymatic trypsin exposure, which is directly associated with cellular dedifferentiation. NP cells are often compared to chondrocytes and, as such, several studies have shown the negative effects of passaging on chondrogenic gene expression (Darling et al., 2005; Lin et al., 2008). The rationale is that non-passaged cells do not need to waste time and energy on perhaps a poor re-differentiation process and, therefore, will be far more efficient in cell based therapy for tissue repair. Previously, we have been able to show that continuous culture can do this for bovine chondrocytes (Rosenzweig et al., 2013; Rosenzweig et al., 2012a) and now for bovine NP cells as well. Although, this strategy alone does not translate into human NP cell phenotype retention. Perhaps, in combination with other culture strategies, such as $3 \mathrm{D}$ cell expansion, this might be beneficial to human NP/disc cell expansion. There are clear regulatory limitations and safety concerns regarding the use of animal products, animal serum and even human serum in clinical stem cell therapies (Halme et al., 2006). As such, there are now commercially available xeno-free and serumfree chemically defined culture media for growth of stem cells and other cell types. We believe that a combination of an enhanced NP-specific xeno-free and serum free medium with passage-free culture may be beneficial for expanding human NP cell populations. Of course, such a formulation must be developed first and then tested. The continuous expansion device is quite different from thermoreversible pNIPAM cell sheets. These innovative sheets are designed to have a very thin layer of thermoreversible material, on which cells can adhere and grow into a dense sheet of cells at $37^{\circ} \mathrm{C}$. Upon cooling to room temperature $\left(\sim 20^{\circ} \mathrm{C}\right)$, the pNIPAM dissociates completely, releasing the connected cell sheet network, which can been layered or rolled for tissue repair applications (Patel et al., 2013). The continuous expansion device constantly applies a small mechanical stimulation to the cells while it physically expands. Cells adhering to a collagen I coating are able to continually proliferate due to increasing dish surface area, while remaining at a near-confluence density. However, the outcome for both techniques may be quite similar since there is little to no need for enzymatic removal of the expanded cell population. A direct comparison of these two techniques in NP phenotype retention would indeed be an interesting study, which could go a long way towards clinical applications of human NP cell therapies.

Zhen Li: What are the reasons and mechanisms behind the different response of bovine and human NP cells to the continuous culture? Is it because the age and degeneration grade of bovine and human NP cells in the study are not comparable? Or is it a real species difference phenomenon? Authors: All bovine donors used in this study were young (18-21 months) and the tissue was pristine. Initially, we assessed human cells isolated from herniation surgical samples, since this may be a feasible cell source for disc implantation therapy. This tissue was from adults with mature discs and potential degenerative changes. We were not that surprised that there was a difference between pristine and degenerate tissue and we thought that the difference may be due to age and state of degeneration. Also, we thought the difference may be due to a mixed cell population from the herniated tissue. To sort this out, we tested pure NP cell preparations from non-degenerate, mature and very young human tissue. The results of continuous expansion were in both cases similar to the more degenerate herniation cells. At this point, we can only speculate about these results, since it is difficult to directly compare age between species. Is 18-21 months bovine tissue comparable to a 15 years old human? If not, then the difference may still be due to age differences. However, this will be hard to resolve, since it is extremely rare that we have tissue from very young human donors. Likewise, it is difficult to have access to older bovine tissue. We speculate that the difference is species specific, perhaps due to different mechanical loading environments of the discs. The lumbar spine in human is exposed to axial loading with some bending and torsion, while the bovine caudal discs are exposed to frequent bending motions with high force during tail movement. We can further speculate that the bovine NP cells are accustomed to and respond in a more favourable way to the tensile strains applied during continuous expansion on the silicone culture dishes. This may not be what human NP cells normally would experience and, therefore, it may have a more negative effect on the human NP cells. Nonetheless, this must be further assessed prior that any definitive conclusions can be drawn. The main take-home message from our study is that there are many factors we still do not know and that it is not possible to directly translate findings between species. 
Laura Creemers: Why would the use of autologous NP cells be required in a confined environment such as the disc? In other words, could we also revert to donor cells? Authors: The central NP region of the adult IVD is mainly avascular in healthy, non-degenerate tissue. Therefore, it is thought to be somewhat immune-privileged. Theoretically, donor cells could be implanted with low-risk of immunereactions within the tissue. However, in degenerate IVDs, the tissue loses proteoglycan content (a natural barrier to nerve/vessel ingrowth) and can become torn and fissured. An inflammatory environment is gradually increased and vessel ingrowth and immune cell infiltration can occur. We can speculate that perhaps this was a consideration taken for the 4 clinical trials, which use autologous stem cells or disc-derived chondrocytes for NP implantation and repair. Also, there is a risk that some cells leak out during implantation and, if that happens, an immune response could be activated. Another potential means of introducing immune cells into the disc may be from the needle injection itself.

\section{Additional References}

Halme DG, Kessler DA (2006) Fda regulation of stemcell-based therapies. N Engl J Med 355: 1730-1735.

Patel NG, Zhang G (2013) Responsive systems for cell sheet detachment. Organogenesis 9: 93-100.

Editor's note: The Scientific Editor responsible for this paper was Mauro Alini. 